\title{
Cytomegalovirus-Associated Splanchnic Vein Thrombosis in Immunocompetent Patients: Two Case Reports and Literature Review
}

\author{
Antonella Tufano, MD, $\mathrm{PhD}^{1}$ Paola Contaldi, MD ${ }^{1}$ \\ Nicoletta Franco, MD ${ }^{1}$ Anna Maria Cerbone, MD ${ }^{1}$ \\ ${ }^{1}$ Department of Clinical Medicine and Surgery, Regional Reference \\ Centre for Coagulation Disorders, "Federico II" University Hospital, \\ Naples, Italy \\ Semin Thromb Hemost 2018;44:298-303.
}

\author{
Antonio Coppola, MD ${ }^{1}$ Assunta Nardo, $\mathrm{MD}^{1}$ \\ Giovanni Di Minno, MD
}

Address for correspondence Antonella Tufano, MD, PhD, Department of Clinical Medicine and Surgery, Regional Reference Centre for Coagulation Disorders, “Federico II” University Hospital, Via S. Pansini, 5, 80131 Naples, Italy (e-mail: atufano@unina.it).

ceptive (OC) use, prolonged immobilization, malignancies, trauma, or inherited thrombophilic conditions. ${ }^{7,8}$

We therefore wish to report two recent cases of young immunocompetent patients referred to our tertiary care center who experienced SVT associated with acute CMV infection. We follow these case descriptions with a brief narrative review focused on the available literature evidence especially with respect to: (1) clinical presentation and risk factors of events, (2) mechanisms involved in the thrombotic process in immunocompetent patients with acute CMV infection, and (3) management of these thrombotic events.

Case I: A 40-year-old man was admitted to our center because of fever, abdominal pain, and diarrhea. He had been treated ineffectively with antibiotic drugs in the previous weeks. His past clinical history included arterial hypertension and obesity, and he was a carrier of $\beta$-thalassemia. On admission, the patient had persistent fever $\left(>38^{\circ} \mathrm{C}\right)$ and pain in the right hip. No superficial lymphadenopathy on physical examination was detected. Blood tests revealed leukopenia with relative lymphocytosis and elevated liver cytolysis indices, as well as increased values of lipase, amylase, lactate dehydrogenase, and D-dimer. Chest X-ray and electrocardiogram were negative. A computed tomography (CT) scan of his abdomen demonstrated an extensive thrombus of splenic vein and superior MVT. The screening for inherited/acquired thrombophilia, including the search for factor V Leiden (FVL) and prothrombin (FII) G20210A gene polymorphisms, the detection of plasma anticardiolipin antibodies and lupus anticoagulant, and measurements of plasma antithrombin, protein $C$, protein $S$, and homocysteine were negative. Hematological (negative bone marrow aspirate and JAK2 mutation) or clear-cut neoplastic diseases were excluded. Serological tests for HIV, hepatitis (A, B, C, and E), toxoplasma, and EBV were normal, whereas CMV serology revealed high-titer immunoglobulin $\mathrm{M}(\operatorname{IgM})$ antibodies (100 published online January 2, 2018
Issue Theme Editorial Compilation V; Guest Editors: Emmanuel J. Favaloro, PhD, FFSc (RCPA), and Giuseppe Lippi, MD.
Copyright $\odot 2018$ by Thieme Medical Publishers, Inc., 333 Seventh Avenue, New York, NY 10001, USA. Tel: +1(212) 584-4662.
DOI https://doi.org/ 10.1055/s-0037-1615262. ISSN 0094-6176. 
$\mathrm{IU} / \mathrm{mL}$, normal values $<12 \mathrm{IU} / \mathrm{mL}$ ) with negative immunoglobulin G ( $\operatorname{IgG}$ ) antibodies, and a low IgG avidity index: 0.122 (reference range $<0.2$ : low), thus suggesting acute infection. The patient was put on anticoagulation with therapeutic doses of low molecular weight heparin (LMWH) (enoxaparin $100 \mathrm{IU} / \mathrm{kg}$ twice daily) and then warfarin. The systemic symptoms gradually resolved without the need for ganciclovir treatment. After 6 months, a complete resolution of the thrombus was shown at the CT scan, and warfarin therapy was discontinued.

Case II: A 24-year-old female presented to the emergency department with fever and abdominal pain for the past week. She was a carrier of $\beta$-thalassemia, was obese, and reported a history of uterine myomas and gallbladder stones. On this basis, a biliary colic was first hypothesized, not confirmed at ultrasound evaluation, while an urgent CT scan of abdomen demonstrated thrombosis of the inferior mesenteric vein, without extension to portal and splenic veins. Use of OCs was denied, hematological and neoplastic causes were excluded, and no other cardiovascular risk factors were present. Serological tests for HIV, hepatitis (A, B, C, and E), toxoplasma, and EBV were negative, whereas CMV serology revealed hightiter IgM antibodies (129 IU/mL, normal values $<12 \mathrm{IU} / \mathrm{mL}$ ) with negative IgG antibodies, consistent with acute infection. She was treated with low-dose LMWH (enoxaparin 4,000 IU once daily) for 2 to 3 weeks. Four years later, the patient was referred to us for counseling about pregnancy, and at this time, a heterozygous FII G20210A polymorphism was identified. A CT scan of her abdomen showed resolution of the previous thrombosis. Due to the presence of thrombophilia and obesity, she received an intermediate-dose enoxaparin prophylaxis (100 IU/kg once daily) during pregnancy, without thromboembolic recurrences.

CMV infection was the most likely precipitating factor for SVT in these two patients because no other cause/disease could explain the clinical and laboratory presentation. In both the cases, fever and abdominal pain were detectable. Anticoagulant therapy, although conducted in the second patient at low dose and only for 2 to 3 weeks, resulted in complete resolution of thrombosis.

- Table 1 summarizes the largest studies and reviews reflecting on the association between CMV infection and SVT. Risk factors and clinical characteristics of hospitalized patients with acute CMV infection-associated thrombosis were evaluated in 2010 in a retrospective case-control study $^{11}$ including 140 consecutive patients (mean age: $37.3 \pm 17.4$ years) diagnosed with the acute infection in a tertiary medical center and 140 matched controls without CMV infection. Among the control group, none of the patients had thrombotic events, whereas among the study group, nine $(6.4 \% ; p=0.003)$ patients had thromboses: five arterial thromboses (four splenic infarcts and one renal infarct) and four venous thromboses (one PE, one lower limb DVT, one upper limb DVT, and one jugular vein thrombosis). Concomitant predisposing conditions for thrombosis (OCs, immobilization, recent surgery), other than the infection, were present in 6/9 (66.6\%) cases. Binary logistic regression analysis showed that acute CMV infection was indepen- dently associated with thrombosis in the whole cohort $(p=0.004)$, and the use of OCs/hormones or pregnancy were independently associated with thrombosis among patients with acute infection $(p=0.043)^{11}$

In another case-control study, five cases of VTE (three DVT and two PE) and active CMV infection were detected in hospitalized patients, all females, aged below 37 years and showing at least one concomitant VTE acquired risk factor (three during OC treatment, one after surgery, and one in puerperium $).{ }^{12}$ In a case-control study that investigated the potential role of $\mathrm{CMV}$ in hospitalized immunocompetent patients, a higher frequency of positive CMV-IgM and CMV-IgG was observed in VTE patients than in controls. ${ }^{13}$ Seven patients with VTE had CMV-IgM positivity (six women and one man); in two cases, the thrombosis occurred during OC therapy and in one case during pregnancy. ${ }^{13}$

Clinical characteristics of patients with CMV-associated thrombosis were also evaluated in a meta-analysis, published in 2011, that included 97 reports. ${ }^{6}$ Overall, 64 immunocompetent patients and 33 immunocompromised patients were described, with a mean age of $39.7 \pm 14.9$ years and a femaleto-male ratio of $1: 1$, although females were prevalent among the immunocompetent cases. The most prevalent clinical presentations of infection were CMV mononucleosis and CMV colitis. The most frequent sites of thrombosis were lower limbs (DVT) and/or pulmonary circulation (PE) (52/97; 53.6\%), followed by SVT (PVT, superior and inferior MVT, colic vein thrombosis) and splenic infarction. The episodes of DVT or PE were more frequent in immunocompromised patients, whereas SVTs were more prevalent among immunocompetent cases. Arterial thromboses (renal infarct, myocardial infarction, digital ischemia) were rare. ${ }^{6}$ In the meta-analysis, the use of OCs, transient/permanent antiphospholipid antibody (APLA) positivity, and FVL heterozygosis were the most common concomitant predisposing conditions for thrombosis. Other predisposing conditions were surgery, active malignancy, and pregnancy. ${ }^{6}$ Interestingly, the prevalence of inherited thrombophilia was significantly higher among immunocompetent patients compared with immunocompromised patients, ${ }^{6}$ thus suggesting a prothrombotic state associated with the condition of immunosuppression of these patients to be sufficient to induce the thrombosis.

Paran et al in 2013 investigated, for the first time, the longterm effect (6-month incidence) on VTE and/or arterial thrombosis in 6,205 outpatients who tested positive for CMV-IgM antibodies in a large health maintenance organization in Israel. ${ }^{14}$ During 6 months of follow-up from index date (the earliest CMV-IgM testing date), the incidence rates of VTE among CMV-IgM seropositive were higher compared with CMV-IgM seronegative patients (odds ratio [OR]: 2.25; $95 \%$ confidence interval $[\mathrm{CI}]: 1.38-3.66 ; p=0.003)$. The CMV-IgM seropositivity was independently associated with VTE in multivariable logistic regression analysis (OR: 2.49; 95\% CI: $1.53-4.06 ; p<0.0001$ ), but it was not associated with arterial thrombosis. ${ }^{14}$

Clinical characteristics of CMV-related thrombosis have been reviewed by Sherman et al, ${ }^{15}$ who described 78 reports concerning 113 patients. The mean age of patients was 


\begin{tabular}{|c|c|c|c|c|c|c|c|}
\hline 焉蒂 & 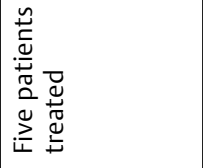 & 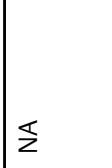 & z & 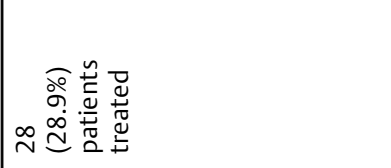 & \& & 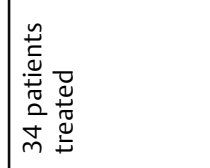 & $\Sigma$ \\
\hline 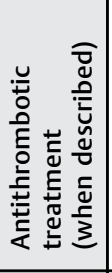 & 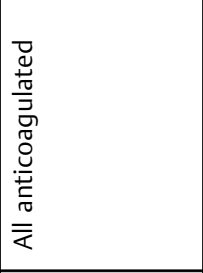 & 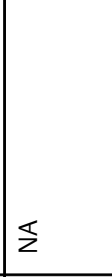 & $\mathbb{z}$ & 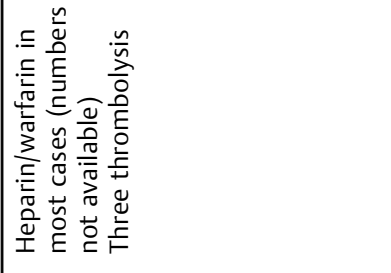 & 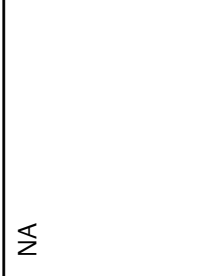 & 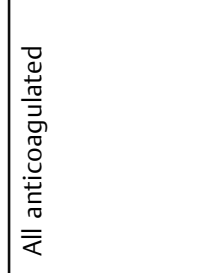 & 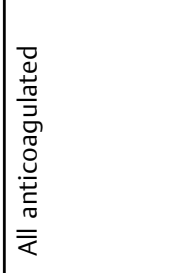 \\
\hline 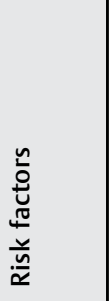 & 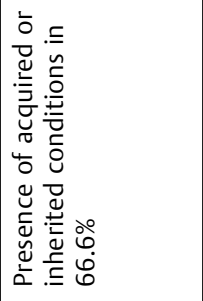 & 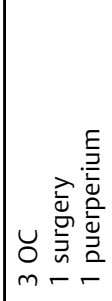 & 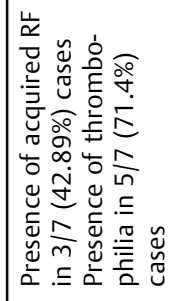 & 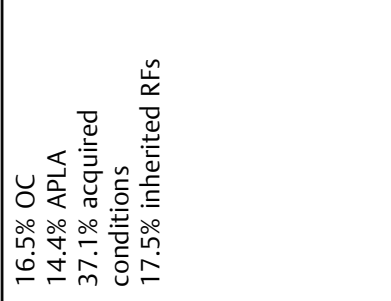 & 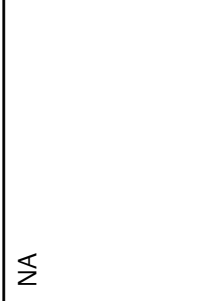 & 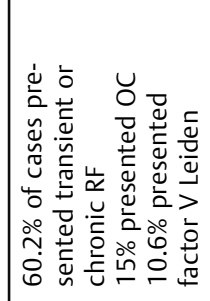 & 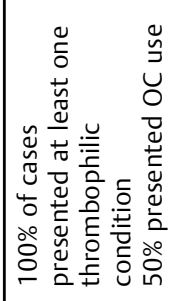 \\
\hline 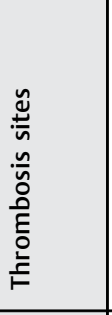 & 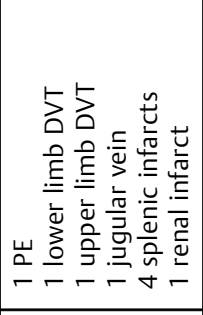 & 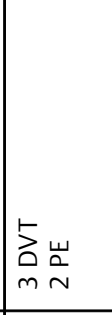 & 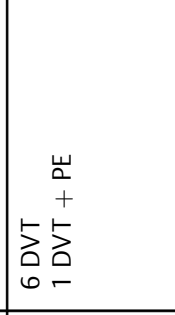 & 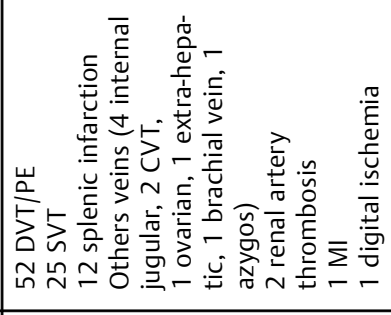 & 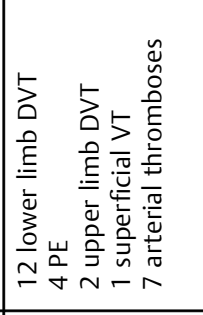 & 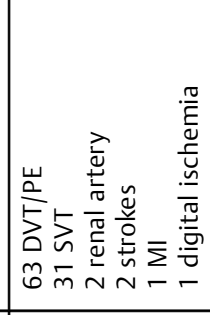 & 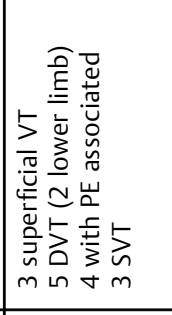 \\
\hline 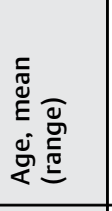 & 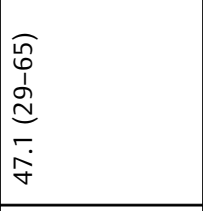 & 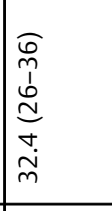 & 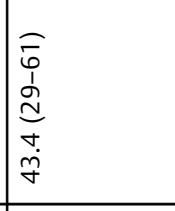 & 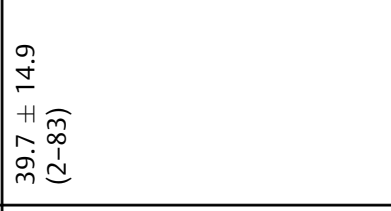 & 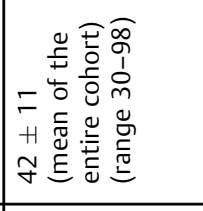 & 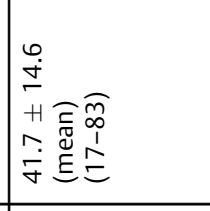 & 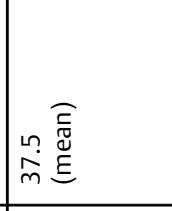 \\
\hline 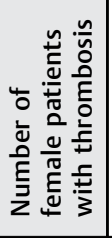 & $\frac{a}{N}$ & $\frac{n}{n}$ & $\frac{1}{6}$ & $\frac{\hat{a}}{\hat{b}}$ & 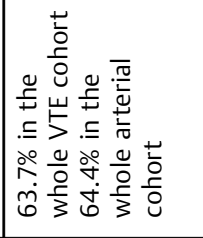 & 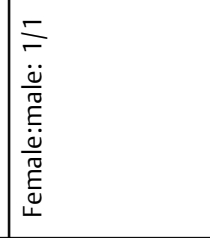 & $\frac{O}{\sigma}$ \\
\hline 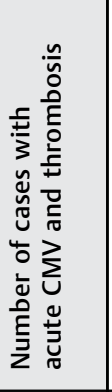 & 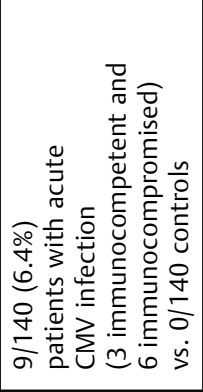 & 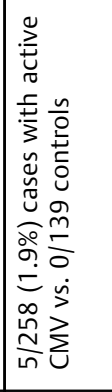 & 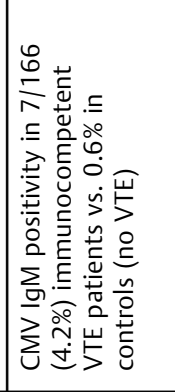 & 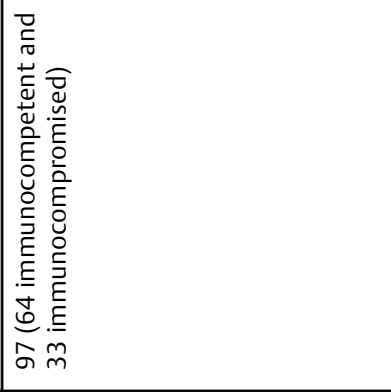 & 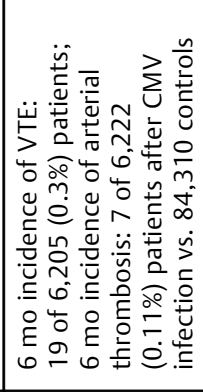 & $\stackrel{m}{F}$ & 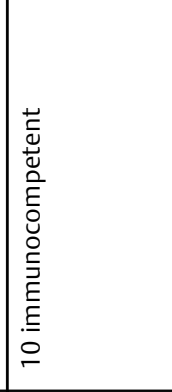 \\
\hline . ज़ & 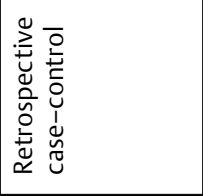 & 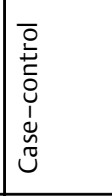 & 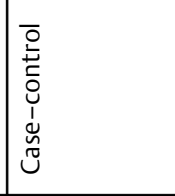 & 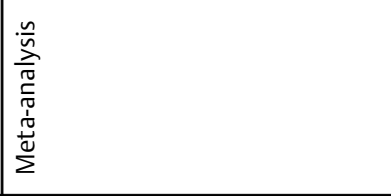 & 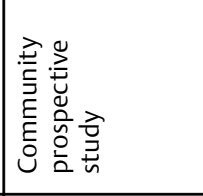 & 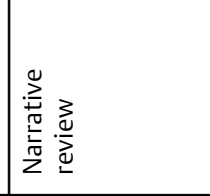 & 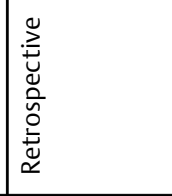 \\
\hline 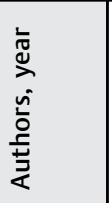 & 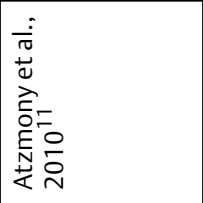 & 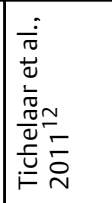 & 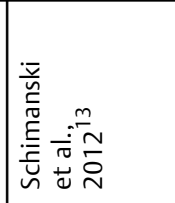 & 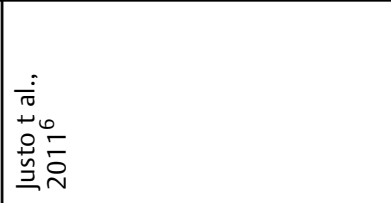 & 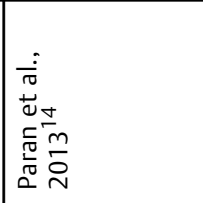 & 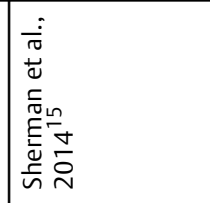 & 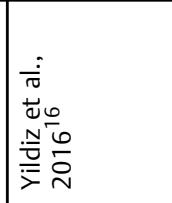 \\
\hline
\end{tabular}




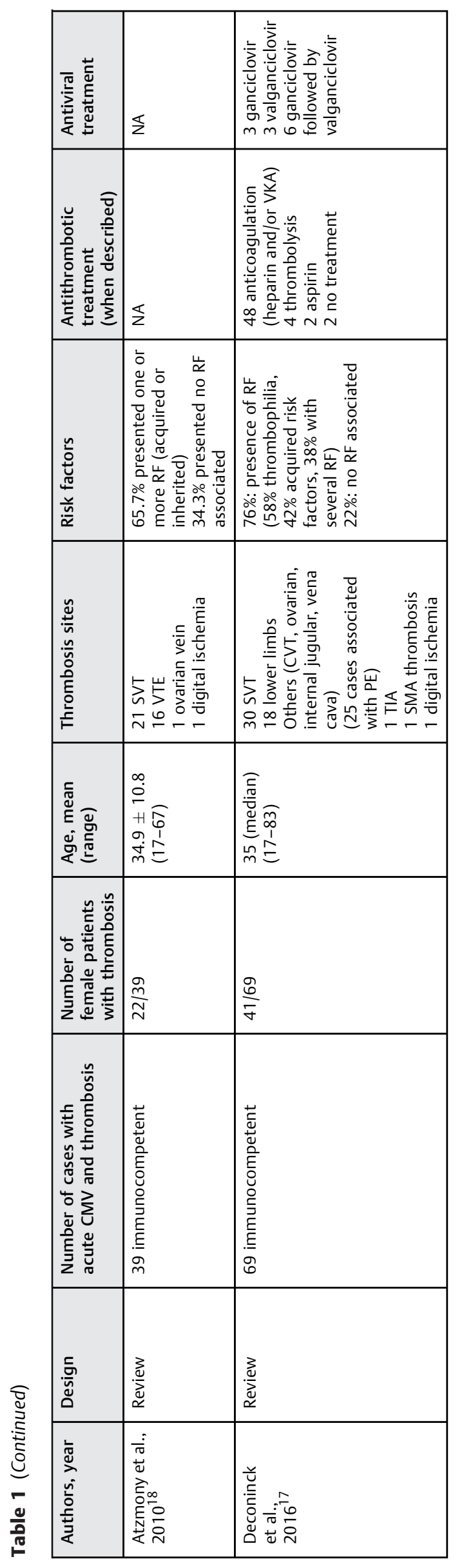

$41 \pm 14.6$ with a female-to-male ratio of $1: 1$. The female-tomale ratio was reanalyzed separately in controlled studies, including consecutive patients, and was $1.6 .^{15}$

Recently, Yildiz et al identified 10 patients with synchronous acute CMV infection among 1,007 consecutive patients with VTE. Patients with coexistent VTE and acute CMV infection were younger ( 37.5 vs. 56.6 years; $p=0.0088$ ) and exhibited a female predominance (90 vs. 56\%; $p=0.026$ ) in comparison to the whole cohort. Hereditary thrombophilia was identified in 9 of 10 patients. Acquired risk factors for VTE such as OCs and pregnancy were equally prevalent in patients with and without CMV infection. ${ }^{16}$

In another recent literature review, ${ }^{17} 49$ papers with a total of 69 patients ( 41 women and 28 men) with VTE complicating CMV primary infection were analyzed. The main sites of venous thrombosis were SVT (30 patients) and DVT of lower limbs (18 patients). One-third of patients presented with PE (25 patients). Forty-nine (76\%) of the 64 patients screened for thrombophilia had at least one VTE risk factor, inherited or acquired thrombophilia for 37 (58\%) patients, and another risk factor for 27 (42\%) patients. ${ }^{17}$ Anticoagulant treatment was described for 48 patients, and treatment duration reported in 20 cases was 3 to 6 months. Anticoagulation was discontinued without any radiological evidence of thrombosis resolution for most patients.

The potential mechanisms of thrombosis in CMV infection deserve mention. Venous thrombosis is a multifactorial disease that can be triggered by environmental factors and inherited predisposition. Based on data reported in the literature, it has been suggested to include CMV infection among VTE risk factors/triggers. ${ }^{4,15,18,19}$ However, unlike immunosuppressed patients, in whom infection is often the only condition associated with thrombosis, inherited thrombophilia and acquired risk factors for thrombosis often coexist in immunocompetent patients with SVT. ${ }^{6,18}$

In a review of case reports and case series on CMVassociated thrombosis in immunocompetent adults, ${ }^{18} 39$ patients were described (mean age: $34.9 \pm 10.8$ years). Most events (21/39; 54\%) were SVT. Overall, 14 (35.9\%) patients had one or more acquired risk factors for thrombosis; 16/35 (45.7\%) patients investigated for inherited thrombophilia had one or more inherited predispositions for thrombosis. Only 12 (34.3\%) patients did not show any acquired or inherited predisposition for thrombosis other than CMV infection. The most common $(n=13 ; 33.3 \%)$ acquired predisposition for thrombosis was the use of OCs,

Table 2 CMV infection and thrombosis: underlying mechanisms

\begin{tabular}{|l|}
\hline Endothelial dysfunction (increased tissue factor exposure) \\
\hline Platelet and leukocyte adhesion \\
\hline Increased tissue factor exposure in infected monocytes \\
\hline Procoagulant properties of CMV envelope \\
\hline Increased factor VIII and von Willebrand factor levels \\
\hline Transient antiphospholipid antibodies \\
\hline
\end{tabular}

Abbreviation: CMV, cytomegalovirus. 
Table 3 Suggested strategies for CMV-associated venous thromboembolism

\begin{tabular}{|l|l|}
\hline Acute CMV infection + no RF & No anticoagulation \\
\hline Acute CMV infection $+\geq 1$ RF & Preventive anticoagulation \\
\hline Acute CMV infection + VT & VKA, INR 2-3, 3 mo \\
\hline Acute CMV infection $+V T+\geq 1$ irreversible RF & VKA, INR 2-3, at least 3 mo, discuss life-long treatment \\
\hline
\end{tabular}

Abbreviations: CMV, cytomegalovirus; INR, international normalized ratio; mo, month; RF, risk factors for venous thromboembolism; VKA, vitamin $\mathrm{K}$ antagonist; VT, vascular thrombosis.

Note: Modified from Deconinck et al. ${ }^{17}$

whereas FVL was the most frequently detected inherited thrombophilia factor $(n=6 ; 17.1 \%)^{18}(-$ Table 1$)$. The relatively higher frequency of SVT rather than DVT/PE found in immunocompetent patients with CMV infection ${ }^{6,18}$ may be explained by a role of acute CMV hepatitis as a local inflammatory predisposing condition. ${ }^{4}$

The mechanisms by which CMV infection may induce thrombosis are varied ( - Table 2 ). The most accepted theories suggest that CMV infects endothelial cells, thus inducing membrane alteration and expression of adhesion molecules causing platelet and leukocyte adhesion and generating tissue factor (TF) exposure, factor $\mathrm{X}$ activation, and thrombin formation. ${ }^{5,19,20}$ Intrinsic CMV envelope procoagulant properties may be another cause of hemostatic imbalance, and furthermore, TF is present on infected monocytes. ${ }^{19}$ Acute CMV infection is reported to increase circulatory levels of von Willebrand factor and factor VIII. ${ }^{5,18,19}$ Finally, acute CMV infection has been associated with transient appearance of APLA. ${ }^{19-24}$

No guideline on management of CMV-associated venous thrombosis is currently available. In particular, there are no agreed recommendations on the duration of anticoagulation and benefit of antiviral therapy. The majority of CMV-associated venous thromboses reported in the literature were treated with LMWH and warfarin, with the duration of oral anticoagulation ranging between 20 days and 9 months. ${ }^{20}$ Therapy was stopped when the resolution of thrombosis was confirmed or, in some cases, on the basis of reduction or absence of APLA. ${ }^{6,20}$

Three months of anticoagulant therapy seems to be the most appropriate strategy since CMV infection is a transient and reversible risk factor, according to the current guidelines of the American College of Chest Physicians. ${ }^{25,26}$ However, the search for inherited thrombophilia and APLA should guide the choice of extended treatment.

The benefit of primary anticoagulant prophylaxis in patients with acute CMV infection is not defined and should be considered in the presence of multiple concomitant prothrombotic conditions (i.e., hospitalized patients with immobilization or a history of VTE). ${ }^{17}$

As regards CMV antiviral therapy, in the recent review by Sherman et $\mathrm{al}^{15} 30.1 \%$ of the total population of patients studied were treated with antiviral agents (ganciclovir or valganciclovir) and $73.5 \%$ of these had viremia diagnosed by CMV-DNA PCR (deoxyribonucleic acid polymerase chain reaction) or antigen assays. ${ }^{15}$ In the meta-analysis by Justo et al, only $17.2 \%$ of immunocompetent patients received antiviral agents compared with $51.5 \%$ in the immunocompromised group. ${ }^{6}$ In the previously cited literature review, ${ }^{17}$ regarding
49 papers with a total of 69 patients with VTE complicating CMV primary infection, only 11 patients (all cases with severe thromboembolic events) received antiviral therapy (ganciclovir and/or valganciclovir). A positive outcome was observed in all patients, with the exception of a case of BCS. The authors suggested that antiviral therapy should be considered for patients presenting with severe VTE, VTE with a negative outcome despite anticoagulation, and severe organ involvement or for patients managed in the intensive care unit. They also suggested a practical antithrombotic strategy for CMVassociated thrombosis ${ }^{17}$ ( - Table 3 ).

In conclusion, the number of reported SVT in patients with acute CMV infections has increased considerably in recent years, mainly due to the growing awareness of the existence of an association between thrombotic events and this infection. The frequency of SVT is higher than that of DVT/PE in immunocompetent patients with CMV-associated thrombosis in whom concomitant acquired/inherited predisposing factors other than the infection are often recognized.

On the basis of literature data, acute CMV infection should be considered as a possible prothrombotic condition and searched for in adult immunocompetent patients with SVT in the presence of symptoms and signs of the infection. The search for concomitant thrombotic risk factors and inherited/acquired thrombophilic conditions may better guide the optimal management strategy in the absence of specific recommendations or guidelines.

\section{References}

1 Tufano A, Di Capua M, Coppola A, et al. The infectious burden in atherothrombosis. Semin Thromb Hemost 2012;38(05):515-523

2 Squizzato A, Gerdes VE. Viral hepatitis and thrombosis: a narrative review. Semin Thromb Hemost 2012;38(05):530-534

3 Epaulard O, Foote A, Bosson JL. Chronic infection and venous thromboembolic disease. Semin Thromb Hemost 2015;41(06): 644-649

4 Squizzato A, Ageno W, Cattaneo A, Brumana N. A case report and literature review of portal vein thrombosis associated with cytomegalovirus infection in immunocompetent patients. Clin Infect Dis 2007;44(02):e13-e16

5 Galli L, Gerdes VEA, Guasti L, Squizzato A. Thrombosis associated with viral hepatitis. J Clin Transl Hepatol 2014;2(04):234-239

6 Justo D, Finn T, Atzmony L, Guy N, Steinvil A. Thrombosis associated with acute cytomegalovirus infection: a meta-analysis. Eur J Intern Med 2011;22(02):195-199

7 Ladd AM, Goyal R, Rosainz L, Baiocco P, DiFabrizio L. Pulmonary embolism and portal vein thrombosis in an immunocompetent adolescent with acute cytomegalovirus hepatitis. J Thromb Thrombolysis 2009;28(04):496-499 
8 Amitrano L, Guardascione MA, Scaglione M, Menchise A, Romano L, Balzano A. Acute portal and mesenteric thrombosis: unusual presentation of cytomegalovirus infection. Eur J Gastroenterol Hepatol 2006;18(04):443-445

9 Rinaldi F, Lissandrin R, Mojoli F, et al. Acute cytomegalovirus infection as a cause of venous thromboembolism. Mediterr J Hematol Infect Dis 2014;6(01):e2014041

10 Thatipelli MR, McBane RD, Hodge DO, Wysokinski WE. Survival and recurrence in patients with splanchnic vein thromboses. Clin Gastroenterol Hepatol 2010;8(02):200-205

11 Atzmony L, Halutz O, Avidor B, et al. Incidence of cytomegalovirus-associated thrombosis and its risk factors: a case-control study. Thromb Res 2010;126(06):e439-e443

12 Tichelaar VY, Sprenger HG, Mäkelburg AB, Niesters BG, KluinNelemans HC, Lijfering WM. Active cytomegalovirus infection in patients with acute venous thrombosis: a case-control study. Am J Hematol 2011;86(06):510-512

13 Schimanski S, Linnemann B, Luxembourg B, et al. Cytomegalovirus infection is associated with venous thromboembolism of immunocompetent adults-a case-control study. Ann Hematol 2012;91(04):597-604

14 Paran Y, Shalev V, Steinvil A, et al. Thrombosis following acute cytomegalovirus infection: a community prospective study. Ann Hematol 2013;92(07):969-974

15 Sherman S, Eytan O, Justo D. Thrombosis associated with acute cytomegalovirus infection: a narrative review. Arch Med Sci 2014;10(06):1186-1190

16 Yildiz H, Zech F, Hainaut P. Venous thromboembolism associated with acute cytomegalovirus infection: epidemiology and predisposing conditions. Acta Clin Belg 2016;71(04):231-234

17 Deconinck L, Flateau C, Pichenot M, et al. Antiviral therapy of primary cytomegalovirus infection with vascular thrombosis in immunocompetent adults. Med Mal Infect 2016;46(02):87-92
18 Atzmony L, Grosfeld A, Saar N, Justo D. Inherited and acquired predispositions for thrombosis in immunocompetent patients with cytomegalovirus-associated thrombosis. Eur J Intern Med 2010;21(01):2-5

19 Squizzato A, Gerdes VEA, Büller HR. Effects of human cytomegalovirus infection on the coagulation system. Thromb Haemost 2005;93(03):403-410

20 Wang T, Kuttikat A, Pulsalkar P, Nanguzgambo A, Bhalara S. Cytomegalovirus-associated portal vein thrombosis in an immunocompetent patient: an underestimated complication. Oxf Med Case Rep 2015;2015(05):294-296

21 Delbos V, Abgueguen P, Chennebault JM, Fanello S, Pichard E. Acute cytomegalovirus infection and venous thrombosis: role of antiphospholipid antibodies. J Infect 2007;54(01):e47-e50

22 Vidal M, Corbin V, Chanet V, et al. Infections associated to severe thrombotic events and antiphospholipid antibodies [in French]. Med Mal Infect 2005;35(11):552-555

23 Labarca JA, Rabaggliati RM, Radrigan FJ, et al. Antiphospholipid syndrome associated with cytomegalovirus infection: case report and review. Clin Infect Dis 1997;24(02):197-200

24 Gharavi AE, Pierangeli SS, Espinola RG, Liu X, Colden-Stanfield M, Harris EN. Antiphospholipid antibodies induced in mice by immunization with a cytomegalovirus-derived peptide cause thrombosis and activation of endothelial cells in vivo. Arthritis Rheum 2002;46(02):545-552

25 Pichenot M, Morell-Dubois S, Flateau C, Deconinck L, Hatron PY, Lambert M. Acute cytomegalovirus infection as a transient risk factor for thrombosis: report of three cases and focus on specific coagulation pathways. Thromb Res 2013;132(01): 145-147

26 Kearon C, Akl EA, Ornelas J, et al. Antithrombotic therapy for VTE disease: CHEST Guideline and Expert Panel Report. Chest 2016; 149(02):315-352 